\title{
Dispatching a 19-Unit Indian Utility System Using a Refined Differential Evolution Algorithm
}

\author{
J. Jasper and T. Aruldoss Albert Victoire \\ Department of Electrical and Electronic Engineering, Anna University, Regional Centre, Coimbatore 641047, India \\ Correspondence should be addressed to J. Jasper; mailtojasper@gmail.com
}

Received 30 June 2013; Accepted 13 December 2013; Published 12 January 2014

Academic Editor: Bijaya Panigrahi

Copyright (C) 2014 J. Jasper and T. A. A. Victoire. This is an open access article distributed under the Creative Commons Attribution License, which permits unrestricted use, distribution, and reproduction in any medium, provided the original work is properly cited.

\begin{abstract}
This paper presents a differential evolution with neighborhood based mutation (DE-NM) technique to solve Dynamic Economic Dispatch (DED) problem with valve point effects and multiple fuel options. A new mutation scheme based on neighborhood topology is presented with an aim to achieve the cost reduction together satisfying the dynamic behavior of the generating units over the considered time period. The neighborhood based mutation (NM) balances the exploration and exploitation of the search effort of differential evolution (DE) technique. The NM method enhances the convergence speed and the performance of the DE technique. The performance of the DE-NM is tested on a 10-unit and a real public Indian utility system with 19 generating units. Both the test systems are illustrated under different load patterns. The dispatch results obtained using the proposed method for the Indian system have considerably reduced the operating cost and optimized its operation.
\end{abstract}

\section{Introduction}

Dynamic economic dispatch (DED) is a vital task in the economic operation of modern power system $[1,2]$. The DED is defined as the optimal allocation of the predicted load profile among the online generators for a specific period of time. The objective of DED is to minimize the total fuel cost over the whole scheduling period subjected to practical and technical constraints. In DED the generation schedule is greatly affected due to ramp rate limits [3]. This ramp rate constraint sets a limit on the rate of change of electrical power output for the safe operation of the generating units. Thus the DED problem is a practical formulation in real-time power system problem.

Actual DED formulation mainly depends on the representation of the fuel cost curve of the generators. Several conventional methods [4-6] such as lambda iteration, base point participation factor, Newton method, gradient method and Dynamic programming (DP) [7] is used to solve the DED problem by assuming the fuel cost curve of the generators piecewise linear and non-monotonically increasing. But, in practical generation the fuel cost curve is non-linear and non-convex because of valve point effect [8], multiple fuel option [9] and operational constraints such as prohibited operating zones [10], spinning reserves [11]. Thus makes the above conventional methods fail to solve for quality solution.

To overcome the drawback in the conventional methods, heuristic techniques were introduced. Recently, artificial intelligence techniques such as Artificial Neural Network (ANN) [12], genetic algorithm (GA) [8], evolutionary programming (EP) [13], simulated annealing (SA) [14], particle swarm optimization (PSO) [15], differential evolution (DE) [16], modified differential evolution (MDE) [17], and hybrid algorithm (HA) $[18,19]$ are widely used to solve the DED problem. All the techniques have yielded success to certain extent, but they suffer from premature convergence, local trapping of the problem, and setting the control parameter.

Combining deterministic techniques with evolutionary algorithms (EAs) is a promising alternative to solve highly nonlinear and nonconvex cost functions. Probabilistic methods do not always guarantee discovering the global optimum solution in finite time since updating their candidate's position in the solution space requires probabilistic rules. Therefore, fine tuning of the above techniques was applied for every improvement in the solution. Plenty of literature work has been done on the fine tuning of above methods. To be 
concise, a few are included in the reference. Hybridization of EP with SQP (EP-SQP) [20], PSO with SQP (PSO-SQP) [21], and chaotic differential evolution with SQP (DEC-SQP) [22] are few examples.

Differential evolution proposed by Storn and Price is one of the EA widely used in solving power system optimization problems. DE has got many advantages such that it is simple and easy to understand, and it can handle integer and discrete optimization, ease of use, fast convergence and robustness. In addition, DE is good at exploring the search space and locating the region of global optimum. Like other EAs, DE performance decreases as search space dimensionality increases. Also, DE is sensitive to the choice of the control parameters and it is difficult to adjust them for different problems [23]. Such contradictions in adjusting the control parameters can be overcome by self-adaptive techniques [24]. Hybridization of DE with other heuristic or local different algorithms is considered as an alternate direction of improvement of classical DE.

From the literature [22-25], it can be observed that the main modifications, improvements, and developments on DE focus on adjusting control parameters in self-adaptive manner and or hybridization with other local search techniques. Very few enhancements have been implemented to modify the standard mutation strategies or to propose new mutation rules so as to enhance the total search ability of DE and to overcome the problems of stagnation or premature convergence [25]. In addition, DE is good at exploring the search space but slow at exploitation of solution [26]. In this paper DE algorithm with a neighborhood based mutation (DE-NM) [27] is developed for solving the DED problem. The new mutation scheme utilizes a concept of neighborhood of each population member. The neighborhood based mutation (NM) balances both the exploration and exploitation process to enhance the search ability of DE.

The significant contribution of this paper is optimum generation schedule of an Indian utility system for three different load patterns. The Indian utility system consists of 19 generating units with the fuel cost function taking into account the valve-point effect and multiple fuel option. The DED problem of the Indian utility system is solved conventionally by priority list method where the generating units are allowed to run at full load to meet the demand. Also, real time constraints, such as ramp rate limits, prohibited operating zones, and so forth, are not taken into account in the solution. In recent years pilot attempts are taken to implement various artificial techniques in the operation and control of Indian power system. One such attempt is presented in this paper to solve the DED problem with valve point loading, ramp rate limits, prohibited operating zones, multiple fuel options, and spinning reserve.

This paper is organized as follows: DED problem is formulated in Section 2. Sections 3 and 4 give a detailed description of the DE-NM. Section 5 describes the implementation of DE-NM to DED problem. Analysis of DENM method with two systems is provided in Section 6 and Section 7 outlines the conclusion.

\section{Problem Formulation}

The objective function of DED problem is to minimize the total production cost of power over a given dispatch period, while satisfying various constraints. The objective function is formulated as

$$
\text { Minimize, } F_{T}=\sum_{h=1}^{H} \sum_{i=1}^{N} F_{i h}\left(P_{i h}\right) \text {. }
$$

Generally, the generator cost function is usually expressed as a quadratic polynomial as

$$
F_{i h}\left(P_{i h}\right)=a_{i} P_{i h}^{2}+b_{i} P_{i h}+c_{i} .
$$

For accurate nonconvex models of the objective function, the DED problem with valve point effects has to be considered by superimposing a rectified sinusoid component in the traditional quadratic fuel cost function as formulated in

$$
F_{i h}\left(P_{i h}\right)=a_{i} P_{i h}^{2}+b_{i} P_{i h}+c_{i}+\left|e_{i} \sin \left(f_{i}\left(P_{i h}^{\min }-P_{i h}\right)\right)\right| .
$$

Similarly practical generating units are supplied with multiple fuel sources and the cost functions of these units are represented with few or several piecewise quadratic functions. Such a cost function is called as a hybrid cost function and each segment of the hybrid cost function gives some information about the fuel burnt. The hybrid cost function is formulated as

$$
\begin{aligned}
& F_{i h}\left(P_{i h}\right) \\
& =\left\{\begin{array}{cll}
a_{i, 1} P_{i h}^{2}+b_{i, 1} P_{i h}+c_{i, 1}, & \text { fuel } 1, & P_{i h}^{\min } \leq P_{i h} \leq P_{i h, 1} \\
a_{i, 2} P_{i h}^{2}+b_{i, 2} P_{i h}+c_{i, 2}, & \text { fuel } 2, & P_{i h, 1}<P_{i h} \leq P_{i h, 2} \\
\vdots & \\
a_{i, k} P_{i h}^{2}+b_{i, k} P_{i h}+c_{i, k}, & \text { fuel } k, & P_{i h, k-1}<P_{i h} \leq P_{i h}^{\max } .
\end{array}\right.
\end{aligned}
$$

For more accurate dispatch results, the valve point effect and the multiple fuel options are integrated into the basic quadratic cost function. Thus the basic quadratic cost function given in (2) with $N$ generating units and $N_{F}$ fuel options for each unit is formulated as

$$
\begin{array}{r}
F_{i h}\left(P_{i h}\right)=a_{i, k} P_{i h}^{2}+b_{i, k} P_{i h}+c_{i, k} \\
+\left|e_{i, k} \sin \left(f_{i, k}\left(P_{i h, k}^{\min }-P_{i h}\right)\right)\right| \\
\text { if } P_{i h, k}^{\min } \leq P_{i h} \leq P_{i h, k}^{\max }, \text { fuel option } k, \\
k=1,2, \ldots, N_{F} .
\end{array}
$$

The objective function as given in (1) is subjected to the following equality and inequality constraints.

The power output from all the generating units must satisfy the total demand and the transmission losses of the system. The equality constraint is formulated as

$$
\sum_{i=1}^{N} P_{i h}=P_{D h}+P_{\text {Loss }, h} .
$$


The transmission loss is expressed in a quadratic form as

$$
P_{\text {Loss }, h}=\sum_{m=1}^{N} \sum_{n=1}^{N} P_{m h} B_{m n} P_{n h} .
$$

The real power output of each generating unit is limited by the maximum and minimum power limit of the units. It is formulated as

$$
P_{i}^{\min } \leq P_{i h} \leq P_{i}^{\max }
$$

The operating range of the generating units is restricted by their ramp rate limits. This is formulated as

$$
\begin{aligned}
& P_{i h}-P_{i(h-1)} \leq \mathrm{UR}_{i} \quad \text { if generation increases, } \\
& P_{i(h-1)}-P_{i h} \leq \mathrm{DR}_{i} \quad \text { if generation decreases. }
\end{aligned}
$$

Thus (8) is modified as

$$
\max \left(P_{i}^{\min }, P_{i(h-1)}-\mathrm{DR}_{i}\right) \leq P_{i h} \leq \min \left(P_{i}^{\max }, P_{i(h-1)}+\mathrm{UR}_{i}\right)
$$

Physical limitations of power plant components restrict the operation of generating units in certain operating regions known as prohibited zones. The power generated by each unit should lie either above or below the prohibited zones. Thus the feasible operating zones for the generating units are formulated as

$$
\begin{gathered}
P_{i}^{\min } \leq P_{i h} \leq P_{i, 1}^{l}, \\
P_{i, j-1}^{u} \leq P_{i h} \leq P_{i, j}^{l} \quad j=2,3, \ldots, n, \\
P_{i, n}^{u} \leq P_{i h} \leq P_{i}^{\max } .
\end{gathered}
$$

The spinning reserve constraint considering the prohibited operating zones is formulated as

$$
\begin{gathered}
\sum_{i=1}^{N} S_{i h} \geq S_{R}, \\
S_{i h}=\left\{\begin{array}{c}
\min \left\{\left(P_{i}^{\max }-P_{i h}\right), S_{i}^{\max }\right\} \\
\text { for units without prohibited zones } \\
0, \quad \text { otherwise. }
\end{array}\right.
\end{gathered}
$$

From (12) it is shown that a unit with prohibited operating zones does not contribute to the spinning reserve of the system. This is because the prohibited zones severely restrict the generating unit's flexibility to regulate system load.

\section{Neighborhood Based Mutation (NM)}

The optimization process in DE involves three basic operations such as mutation, crossover, and selection. Mutation in $\mathrm{DE}$ is a special kind of differential operator which is used to generate mutant or donor vectors. Actually it is the process of mutation, which demarcates one DE scheme from another. The rate of convergence of DE as well as its accuracy can be improved largely by applying different mutation strategies. Storn and Price proposed many DE variants applicable to different problems [28]. In this paper a new DE variant, based on neighborhood topology of the parameter vectors, is presented to improve the convergence characteristics of DE [29]. This concept of neighborhood topology is borrowed from the PSO algorithm.

3.1. Neighborhood Model. A graph of interconnection of vectors is called as neighborhood structure. The vectors in the neighborhood structure are assumed to be arranged in a circular fashion. Consider a DE population $P=$ $\left[\vec{X}_{1}, \vec{X}_{2}, \ldots, \vec{X}_{\mathrm{NP}}\right]$ where each, $\vec{X}_{i}$ is a $D$-dimensional parameter vector. For every $\vec{X}_{i}$ a neighborhood of radius $K$ is defined which consists of vectors $\vec{X}_{i-K}, \ldots \vec{X}_{i}, \ldots, \vec{X}_{i+K} \cdot K$ is a nonzero integer from 0 to $(\mathrm{NP}-1) / 2$. A proper balance between exploration and exploitation is necessary for efficient and effective operation of DE. Two kinds of neighborhood mutation models are presented to control the exploration and the exploitation process. One is local mutation model which has a greater tendency to locate the optimal solution of the objective function but needs more iteration. Second is global mutation model which rapidly converges to the optimal solution of the objective function but suffers from premature convergence problem.

3.1.1. Local Neighborhood Mutation Model. For each vector $\vec{X}_{i}$ of the population, a local donor vector is created by employing the best vector in the neighborhood of that member. In this mutation model each vector is mutated using the best position found so far in the neighborhood of it and not in the entire population. The local donor vector $\vec{L}_{i, G}$ is created as

$$
\vec{L}_{i, G}=\vec{X}_{i, G}+\alpha \cdot\left(\vec{X}_{n_{-} \text {best }_{i}, G}-\vec{X}_{i, G}\right)+\beta \cdot\left(\vec{X}_{p, G}-\vec{X}_{q, G}\right),
$$

where the subscript $n$ _best ${ }_{i}$ indicates the best vector in the neighborhood of $\vec{X}_{i, G}$ and $\vec{X}_{p, G}$ and $\vec{X}_{q, G}$ are two randomly chosen vectors from the same neighborhood, that is, $p, q \in$ $[i-k, i+k]$ with $p \neq q \neq i$.

3.1.2. Global Neighborhood Mutation Model. Similarly, the global donor vector is created by using the best vector of the entire population. The global donor vector $\vec{g}_{i, G}$ is created as

$$
\vec{g}_{i, G}=\vec{X}_{i, G}+\alpha \cdot\left(\vec{X}_{g_{-} \text {best }, G}-\vec{X}_{i, G}\right)+\beta \cdot\left(\vec{X}_{r 1, G}-\vec{X}_{r 2, G}\right),
$$

where the subscript $g_{\text {_best }}$ indicates the best vector in the entire population at generation $G$ and $\vec{X}_{r 1, G}$ and $\vec{X}_{r 2, G}$ are two randomly chosen vectors from the population, that is, $r_{1}, r_{2} \in[1, \mathrm{NP}]$ with $r_{1} \neq r_{2} \neq i$.

The local mutation model favours exploration since all the vectors of the population are biased by different individuals. Global mutation model favours exploitation since all the vectors of the population are biased by the same individual. 
The two mutation operators are then combined using a new parameter called weight factor $w \in(0,1)$ to form the actual mutation model of the presented DE algorithm.

$$
\vec{V}_{i, G}=w \cdot \vec{g}_{i, G}+(1-w) \cdot \vec{L}_{i, G}
$$

\section{DE with Neighborhood Based Mutation}

DE begins with a population of NP D-dimensional parameter vectors representing the candidate solutions. The $i$ th vector of the population at the current generation is given as

$$
\vec{X}_{i, G}=\left[x_{1, i, G}, x_{2, i, G}, \ldots, x_{D, i, G}\right] \text {. }
$$

The vector $\vec{X}_{i, G}$ is known as target or parent vector. $G$ represents the subsequent generation and it is given by $G=1,2, \ldots, G_{\max }$. The initial population (i.e., $G=0$ ) is randomly created by covering the entire search space as much as possible within prescribed minimum and maximum bounds. The $j$ th component of the $i$ th target vector is created as

$$
x_{j, i, 0}=x_{j, \min }+\operatorname{rand}(0,1) \cdot\left(x_{j, \max }-x_{j, \min }\right) \text {, }
$$

where $\operatorname{rand}(0,1)$ is a uniformly distributed random number lying between 0 and 1 and is obtained independently for each component of the $i$ th vector. Each target vector of the population is subjected to mutation, crossover, and selection which are explained in the following subsections.

4.1. Mutation. After initialization, DE creates a donor vector $\vec{V}_{i, G}$ corresponding to each target vector $\vec{X}_{i, G}$ in the current generation through mutation. In this method the actual donor vector is created with the help of global and neighborhood mutation models.

For each member a global and local donor vector is created using (13) and (14). After creating the global and local donor vector they are combined using a weight factor $w$ using (15).

4.2. Crossover. In crossover operation few components of the donor vector are exchanged with target vector to form a trial vector $\vec{U}_{i, G}=\left[u_{1, i, G}, u_{2, i, G}, \ldots, u_{D, i, G}\right]$. Two kinds of crossover schemes-exponential and binomial crossoverscan be used in DE. In this paper, binomial crossover is performed on each of the $D$ variables whenever a randomly picked number between 0 and 1 is less than or equal to the $\mathrm{Cr}$ value. The number of parameters inherited from the donor has a binomial distribution. The scheme is outlined as

$$
u_{j, i, G}= \begin{cases}v_{j, i, G}, & \text { if }\left(\operatorname{rand}_{i, j}(0,1) \leq \mathrm{Cr}\right) \\ x_{j, i, G}, & \text { otherwise, }\end{cases}
$$

where $\operatorname{rand}_{i, j}(0,1) \in[0,1]$ is a uniformly distributed random number for each $j$ th component of the $i$ th parameter vector.

4.3. Selection. To keep the population size constant over subsequent generations, the next step of the algorithm calls for selection to determine whether the target or the trial vector survives to the next generation, that is, at $G=G+1$.

The selection operation is given as

$$
\vec{X}_{i, G+1}= \begin{cases}\vec{U}_{i, G}, & \text { if } f\left(\vec{U}_{i, G}\right) \leq f\left(\vec{X}_{i, G}\right), \\ \vec{X}_{i, G}, & \text { if } f\left(\vec{U}_{i, G}\right) \geq f\left(\vec{X}_{i, G}\right),\end{cases}
$$

where $f\left(\vec{X}_{i}\right)$ is the function to be minimized. So if the new trial vector yields an equal or lower value of the objective function than that of target vector, then it replaces the corresponding target vector in the next generation. Otherwise the target vector is retained in the next generation. Hence the population either gets better (with respect to the minimization of the objective function) or remains the same in fitness status but never deteriorates.

The three operations are repeated until a stopping criterion is met which is usually the maximum generation, that is, $G_{\max }$.

\section{Implementation of DE-NM for DED Problem}

Step 1 (initialization of the population). For a population of size NP and dimension $D$, an initial vector (target vector) $X_{i j, G}$ is randomly generated. $D$ represents the number of decision variables to be optimized. In DED problem $D$ represents the number of generating units (i.e., $N$ ) to be considered. The vector $X_{i j}$ is the real power output (i.e., $P_{i j}$ ) of $j$ th unit of the $i$ th population randomly generated within the operating limits using (8). The population is represented in a matrix form as

$$
\text { Population }=\left[\begin{array}{ccccc}
\vec{X}_{11} & \vec{X}_{12} & \vec{X}_{13} & \cdots & \vec{X}_{1 D} \\
\vec{X}_{21} & \vec{X}_{22} & \vec{X}_{23} & \cdots & \vec{X}_{2 D} \\
\vdots & \vdots & & & \vdots \\
\vec{X}_{\mathrm{NP} 1} & \vec{X}_{\mathrm{NP} 2} & & \cdots & \vec{X}_{\mathrm{NPD}}
\end{array}\right] .
$$

Each component of the $i$ th individual in the population is randomly generated such that the component is uniformly distributed within the minimum and maximum power limits of the generating units. The generation of the $j$ th component of the $i$ th individual is given by (17).

Step 2 (handling the generation limit constraints). In Step 1 the power output from each generator is limited to be within minimum and maximum power limits. For the generator with ramp rate limits, the minimum and the maximum power limits are adjusted by using (10). Also, during the recombination and mutation operation, the power output of a generator can go above or below the maximum and minimum limits. Therefore, to restrict the $P_{i}$ to be within the generating limit, a strategy is defined as follow:

$$
P_{i j}= \begin{cases}P_{i}^{\min } & \text { if } P_{i j}<P_{i}^{\min } \\ P_{i}^{\max } & \text { if } P_{i j}>P_{i}^{\max } \\ P_{i j} & \text { otherwise. }\end{cases}
$$


Step 3 (mutation operation). Consider the following.

Step 3.1. A neighborhood structure of radius $K$ is created for each $X_{i j}$ vector.

Step 3.2 (local neighborhood model). In this model a donor vector is created using the best position found so far in a small neighborhood of it and not in entire population. The mutated vector $\vec{L}_{i, G}$ is known as local donor vector and it is obtained using (13).

Step 3.3 (global neighborhood model). In this model a globally best vector $X_{\text {best, },}$ of the entire population is used for mutating a population member. The global donor vector $\vec{g}_{i G}$ is obtained using (14).

Both the local and global donor vectors are combined using (15) to obtain the actual donor vector $V_{i, G}$ of the presented technique.

Step 4 (recombination (crossover)). Recombination is employed to generate a trial vector $U_{i}$ by replacing certain components of $X_{i}$ with corresponding components of donor vector $V_{i}$. The trial vector by crossover operation is obtained using (18).

Step 5 (evaluation of fitness function). The evaluation function is computed from (1) and the generator cost function $F_{i}\left(P_{i}\right)$ used is the cost function considering the valve point effect and multiple fuel option as given in (5). The power balance and the prohibited operating zone constraints are included in the evaluation function by adding a penalty term to the objective function. The power balance constraint is considered without including the transmission losses for simplicity.

Consider

$$
\begin{aligned}
f\left(P_{i h}\right)= & \sum_{i=1}^{N} F_{i h}\left(P_{i h}\right) \\
& +\lambda \cdot\left[\sum_{i=1}^{N} P_{i h}-P_{D h}\right]^{2}+\gamma \cdot\left[\sum_{i=1}^{n} v_{i}^{R}\right],
\end{aligned}
$$

where $\lambda$ is the penalty parameter for not satisfying the load demand and $\gamma$ represents the penalty for a unit loading falling within a prohibited operating zone. $v_{i}^{R}$ is the violation of the prohibited zone constraint for the $i$ th unit which is defined as

$$
v_{i}^{R}= \begin{cases}1, & \text { if } P_{i} \text { violates the prohibited zones } \\ 0 & \end{cases}
$$

Step 6 (selection). Members to constitute the population of next generation $(G+1)$ are decided by $(14)$. The new vector $X_{i,(G+1)}$ is selected based on the comparison of fitness value of both $X_{i}$ and $U_{i}$.

Step 7 (verification of stopping criterion). Set the generation count $G=G+1$. Go to Step 3 until stopping criterion is met which is usually maximum generation count $G_{\max }$.

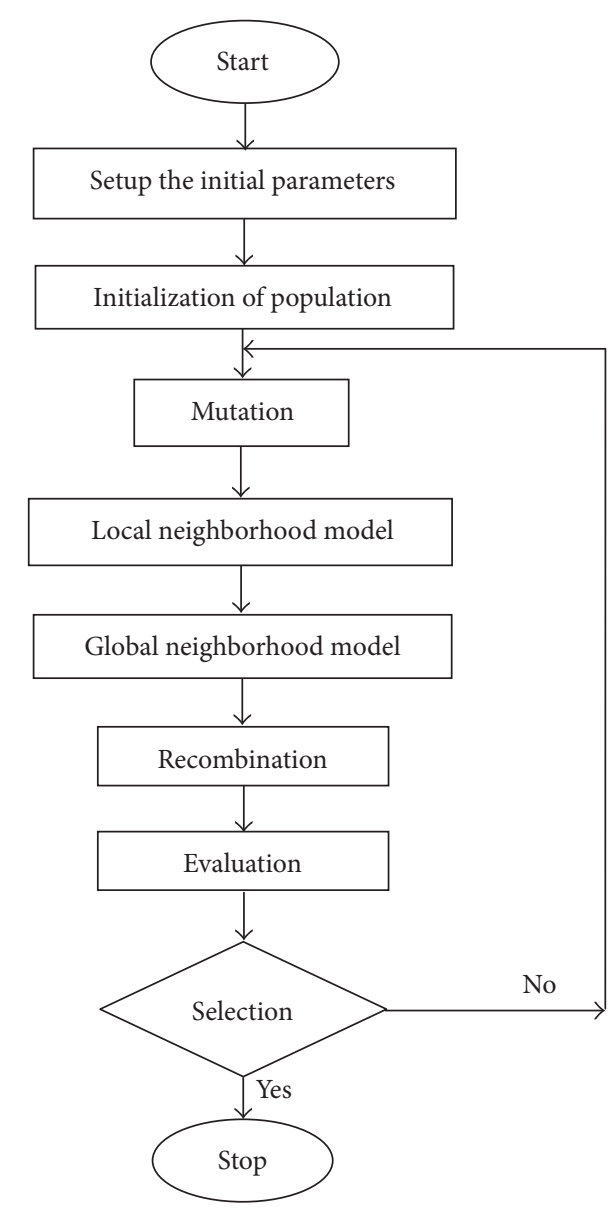

FIGURE 1: Flowchart for DE-NM approach for DED.

\subsection{Flowchart for DE-NM Approach for DED. See Figure 1.}

\section{Test Results and Analysis}

6.1. Description of the Test Systems. In this section two case systems are studied using DE-NM method. The fuel cost functions of the two systems are nonconvex considering valve point effect and multiple fuel option in its fuel cost.

Three different load patterns are applied for both these systems to demonstrate the robustness of the proposed solution technique as well as to show the effectiveness in scheduling for different load patterns (situations). The simulation horizon for all the three load patterns is taken as $24 \mathrm{hr}$ with a dispatch interval of $1 \mathrm{hr}$. The coding is written using the MATLAB 7 programming language and executed in a personal computer machine.

6.2. Parameter Selection. DE-NM consists of four new parameters in addition to $F$ and $\mathrm{CR}$ as in classical DE. They are $\alpha, \beta, w$, and $K$. The role of $\alpha$ and $\beta$ same as that of $F$. Thus, the number of parameters reduced by considering $\alpha=\beta=F$. The most important parameter in DE-NM is the weight factor $w$, which controls the balance between the exploration and exploitation capabilities. To obtain the balance, value of $w$ 
TABLE 1: Best power generation schedule obtained by DE-NM for case study 1.

\begin{tabular}{|c|c|c|c|c|c|c|c|c|c|c|}
\hline \multirow{2}{*}{ Hour } & \multicolumn{10}{|c|}{ Unit } \\
\hline & $1(\mathrm{MW})$ & $2(\mathrm{MW})$ & 3 (MW) & $4(\mathrm{MW})$ & 5 (MW) & $6(\mathrm{MW})$ & 7 (MW) & 8 (MW) & 9 (MW) & 10 (MW) \\
\hline 1 & 168.862 & 177.000 & 250.000 & 250.000 & 240.000 & 166.000 & 250.000 & 180.570 & 243.000 & 250.000 \\
\hline 2 & 163.886 & 177.000 & 250.000 & 250.000 & 234.691 & 163.000 & 250.000 & 173.854 & 253.000 & 250.000 \\
\hline 3 & 165.705 & 177.000 & 250.000 & 250.000 & 235.047 & 160.543 & 250.000 & 178.591 & 253.000 & 250.000 \\
\hline 4 & 165.826 & 177.000 & 250.000 & 250.000 & 240.000 & 163.000 & 250.000 & 181.576 & 249.509 & 250.000 \\
\hline 5 & 172.019 & 177.000 & 250.000 & 250.000 & 457.000 & 150.375 & 250.000 & 183.538 & 252.999 & 247.372 \\
\hline 6 & 192.564 & 177.000 & 269.658 & 265.000 & 451.287 & 196.324 & 250.000 & 249.687 & 302.656 & 254.658 \\
\hline 7 & 181.538 & 177.000 & 253.465 & 253.465 & 446.999 & 180.580 & 250.000 & 246.700 & 218.376 & 250.000 \\
\hline 8 & 172.014 & 177.000 & 250.000 & 250.000 & 457.000 & 163.000 & 250.000 & 176.040 & 249.509 & 250.000 \\
\hline 9 & 171.935 & 177.000 & 250.000 & 250.000 & 240.000 & 153.000 & 250.000 & 183.521 & 253.000 & 250.000 \\
\hline 10 & 170.438 & 177.000 & 250.000 & 250.000 & 240.000 & 163.000 & 250.000 & 176.607 & 253.000 & 246.927 \\
\hline 11 & 179.939 & 177.000 & 253.528 & 253.528 & 457.000 & 174.322 & 250.000 & 191.455 & 273.573 & 250.000 \\
\hline 12 & 165.807 & 177.000 & 250.000 & 250.000 & 234.616 & 163.000 & 250.000 & 180.585 & 251.231 & 247.169 \\
\hline 13 & 178.362 & 177.000 & 251.413 & 251.413 & 457.000 & 161.753 & 250.000 & 190.465 & 270.278 & 250.000 \\
\hline 14 & 205.211 & 177.000 & 250.000 & 250.000 & 457.000 & 163.000 & 282.078 & 254.494 & 345.626 & 264.559 \\
\hline 15 & 205.220 & 177.000 & 310.646 & 265.000 & 457.000 & 224.225 & 277.774 & 254.494 & 406.509 & 273.310 \\
\hline 16 & 194.144 & 177.000 & 274.350 & 265.000 & 457.000 & 199.127 & 262.903 & 250.463 & 304.055 & 246.630 \\
\hline 17 & 178.354 & 177.000 & 261.510 & 261.510 & 457.000 & 162.985 & 250.000 & 247.775 & 266.450 & 246.924 \\
\hline 18 & 167.277 & 177.000 & 250.000 & 250.000 & 233.617 & 163.000 & 250.000 & 179.579 & 253.000 & 246.382 \\
\hline 19 & 163.876 & 177.000 & 250.000 & 250.000 & 451.287 & 163.000 & 250.000 & 177.557 & 248.712 & 250.000 \\
\hline 20 & 171.953 & 177.000 & 250.000 & 250.000 & 235.047 & 160.543 & 250.000 & 184.512 & 253.000 & 250.000 \\
\hline 21 & 168.889 & 177.000 & 250.000 & 250.000 & 234.691 & 163.000 & 250.000 & 181.563 & 253.000 & 250.000 \\
\hline 22 & 178.361 & 177.000 & 252.512 & 252.512 & 240.000 & 171.466 & 250.000 & 192.440 & 263.232 & 246.630 \\
\hline 23 & 165.864 & 177.000 & 250.000 & 250.000 & 240.000 & 156.074 & 250.000 & 183.479 & 253.000 & 246.925 \\
\hline 24 & 176.904 & 177.000 & 250.000 & 250.000 & 457.000 & 158.553 & 250.000 & 181.986 & 251.996 & 250.000 \\
\hline
\end{tabular}

is usually selected from the range $[0,1]$. It is adapted online during the execution of the algorithm by a linear increment scheme; that is, it is initialized with 0 and increased up to 1. Since $w$ starts with 0 , it favors the exploration process during the initial stage and on getting promoted to 1 it favors the exploitation process. The linear increment scheme is given by

$$
w_{G}=\frac{G}{G_{\max }} .
$$

In this paper, the neighborhood size equal to $10 \%$ of the total population size of 100 is considered. The value for both $\alpha$ and $\beta$ is taken as 0.8 and crossover rate $\mathrm{Cr}=0.9$. The DE$\mathrm{NM}$ is applied to both the case studies for 30 independent trials (1000 iterations per trial) with the selected parameters.

6.3. Case Study 1. In this test case, a 10-unit system is optimized to meet three different load patterns. The level of fluctuation of the three different load patterns is given in Figure 2. The peak demand for load pattern 1, pattern 2, and pattern 3 is $3208 \mathrm{MW}, 2460 \mathrm{MW}$, and $3210 \mathrm{MW}$, respectively. The system data and the related constraints for this case study are given in [30]. The complete generation schedule for load pattern 2 corresponding to the minimum generation cost obtained by DE-NM is presented in Table 1 . Table 2 gives the fuel type chosen by the units for the corresponding best generation schedule obtained. The fuel chosen by the units

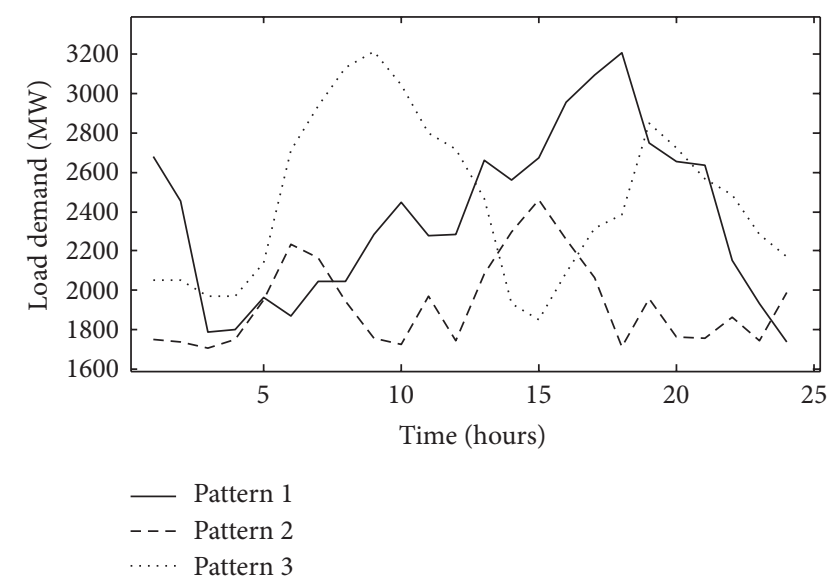

FIGURE 2: Load demand patterns for case study 1.

for all three load patterns is given in Table 2. The table format represents the fuel type chosen by an unit for pattern 1 and it is followed by fuel type for the same unit for patterns 2 and 3, respectively.

The best cost obtained by the DE-NM method considering spinning reserve for pattern 1 is $\$ 10649.68$, for pattern 2 is $\$ 8103.326$, and for pattern 3 is $\$ 11054.83$. The worst cost obtained by the DE-NM method for pattern 1 is 
TABLE 2: Fuel switching of generators for case study 1.

\begin{tabular}{|c|c|c|c|c|c|c|c|c|c|c|}
\hline \multirow{2}{*}{ Hour } & \multicolumn{10}{|c|}{ Unit } \\
\hline & 1 & 2 & 3 & 4 & 5 & 6 & 7 & 8 & 9 & 10 \\
\hline 1 & $2,1,1$ & $1,1,1$ & $1,1,1$ & $3,3,2$ & $3,3,1$ & $3,1,1$ & $2,1,1$ & $3,2,2$ & $3,1,1$ & $1,1,1$ \\
\hline 2 & $2,1,1$ & $1,1,1$ & $1,1,1$ & $3,3,2$ & $3,3,1$ & $3,1,1$ & $1,1,1$ & $3,3,2$ & $3,1,1$ & $1,1,1$ \\
\hline 3 & $1,1,1$ & $1,1,1$ & $1,1,1$ & $2,3,2$ & $1,3,1$ & $1,1,1$ & $1,1,1$ & $2,2,2$ & $1,1,1$ & $1,1,1$ \\
\hline 4 & $1,1,1$ & $1,1,1$ & $1,1,1$ & $2,2,2$ & $1,3,1$ & $1,1,1$ & $1,1,1$ & $2,2,2$ & $1,1,1$ & $1,1,1$ \\
\hline 5 & $1,1,1$ & $1,1,1$ & $1,1,1$ & $3,2,2$ & $1,3,3$ & $1,1,1$ & $1,1,1$ & $3,3,2$ & $1,1,1$ & $1,1,1$ \\
\hline 6 & $1,1,2$ & $1,1,1$ & $1,1,1$ & $3,3,3$ & $1,3,3$ & $1,3,1$ & $1,1,1$ & $2,3,3$ & $1,3,1$ & $1,1,1$ \\
\hline 7 & $1,1,2$ & $1,1,1$ & $1,3,1$ & $3,3,3$ & $3,3,3$ & $1,3,1$ & $1,3,1$ & $2,3,3$ & $1,3,1$ & $1,1,1$ \\
\hline 8 & $1,1,2$ & $1,1,1$ & $1,2,1$ & $2,3,2$ & $3,3,3$ & $1,3,1$ & $1,3,1$ & $2,3,2$ & $1,3,1$ & $1,2,1$ \\
\hline 9 & $2,1,2$ & $1,1,1$ & $1,2,1$ & $3,3,2$ & $1,3,1$ & $3,3,1$ & $1,3,1$ & $3,3,2$ & $3,3,1$ & $1,2,1$ \\
\hline 10 & $2,1,2$ & $1,1,1$ & $1,2,1$ & $3,3,2$ & $3,3,1$ & $3,3,1$ & $1,3,1$ & $3,3,2$ & $1,3,1$ & $1,3,1$ \\
\hline 11 & $2,1,2$ & $1,1,1$ & $1,1,1$ & $3,3,1$ & $3,3,3$ & $3,3,1$ & $1,3,1$ & $3,3,2$ & $1,3,1$ & $1,1,1$ \\
\hline 12 & $2,1,2$ & $1,1,1$ & $1,1,1$ & $3,3,2$ & $3,3,1$ & $3,3,1$ & $1,1,1$ & $3,3,2$ & $1,3,1$ & $1,1,1$ \\
\hline 13 & $2,1,2$ & $1,1,1$ & $1,1,1$ & $3,3,3$ & $3,3,3$ & $3,3,1$ & $1,1,1$ & $3,3,2$ & $3,3,1$ & $1,1,1$ \\
\hline 14 & $2,2,1$ & $1,1,1$ & $1,1,1$ & $3,2,3$ & $3,3,3$ & $3,1,1$ & $1,1,1$ & $3,2,3$ & $3,1,1$ & $1,1,1$ \\
\hline 15 & $2,2,1$ & $1,1,1$ & $1,1,1$ & $3,3,3$ & $3,1,3$ & $3,3,3$ & $1,1,1$ & $3,2,3$ & $3,1,1$ & $1,1,1$ \\
\hline 16 & $2,1,1$ & $1,1,1$ & $2,1,1$ & $3,2,3$ & $3,3,3$ & $3,1,1$ & $2,1,1$ & $3,3,3$ & $3,1,1$ & $1,1,1$ \\
\hline 17 & $2,1,1$ & $1,1,1$ & $2,1,1$ & $3,3,2$ & $3,3,3$ & $3,3,1$ & $3,1,1$ & $3,3,3$ & $3,1,1$ & $1,1,1$ \\
\hline 18 & $2,1,2$ & $1,1,1$ & $2,1,1$ & $3,3,2$ & $3,3,1$ & $3,3,1$ & $3,1,1$ & $3,3,2$ & $3,1,1$ & $2,1,1$ \\
\hline 19 & $2,1,2$ & 11,1 & $1,3,1$ & $2,3,2$ & $3,3,3$ & $3,3,1$ & $2,3,1$ & $3,3,2$ & $3,3,1$ & $1,1,1$ \\
\hline 20 & $2,1,2$ & $1,1,1$ & $1,1,1$ & $2,3,2$ & $3,3,1$ & $3,3,1$ & $1,1,1$ & $3,3,2$ & $3,3,1$ & $1,1,1$ \\
\hline 21 & $2,1,2$ & $1,1,1$ & $1,1,1$ & $2,3,2$ & $3,3,1$ & $3,3,1$ & $1,1,1$ & $3,3,2$ & $3,3,1$ & $1,1,1$ \\
\hline 22 & $1,1,2$ & $1,1,1$ & $1,1,1$ & $2,3,3$ & $3,3,1$ & $1,3,1$ & $1,1,1$ & $3,3,2$ & $1,3,1$ & $1,1,1$ \\
\hline 23 & $1,1,1$ & $1,1,1$ & $1,1,1$ & $2,3,2$ & $1,3,1$ & $1,3,1$ & $1,1,1$ & $3,3,2$ & $1,1,1$ & $1,1,1$ \\
\hline 24 & $1,1,1$ & $1,1,1$ & $1,1,1$ & $2,3,2$ & $1,3,3$ & $1,1,1$ & $1,1,1$ & $2,3,2$ & $1,1,1$ & $1,1,1$ \\
\hline
\end{tabular}

$\$ 11952.07$, pattern 2 is $\$ 9163.973$, and pattern 3 is $\$ 12036.89$. The average cost of pattern 1, pattern 2, and pattern 3 for all the 30 trials is $\$ 11451.82$, $\$ 9711.448$, and $\$ 11497.85$. Figures 3(a), 3(b), and 3(c) shows the distribution of the cost obtained for load patterns 1, 2, and 3, respectively. A straight line passing through each distribution area denotes the average cost. The percentage of producing quality solutions by DE-NM is above $60 \%$ as seen from Figure 4 .

6.4. A Short Overview of the Indian Utility System. Indian power system is a geographically dispersed network of generators. For ease of operation, the entire Indian power system has been divided into five regions, namely, Northern Region (NR), Western Region (WR), Southern Region (SR), Eastern Region (ER), and North Eastern Region (NER). While NR, WR, ER, and NER are interconnected as a single grid, the SR had not been interconnected with the national grid. This prevents the SR from receiving benefits from national power market. The SR covering 6, 51,000 Sq. Km of area comprises the states of Andhra Pradesh, Karnataka, Kerala, Pondicherry and Tamil Nadu. The Indian utility system considered in this paper belongs to the state of Tamil Nadu. The Electricity Board of Tamil Nadu state was recently restructured and the board is in the move of handing over transmission and distribution to the private operators.
The South Indian network comprises of 19 thermal units, located at various parts of the state of Tamil Nadu. Since the system under consideration belongs to the state department, the technical data of the system cannot be published is available with the corresponding author, and will be provided on request. Also the data of system network is approximated neglecting several local feeder data this work neglects estimation of transmission losses for simplicity. Even then, as per the board's advice, this work assumes a uniform $20 \%$ loss in the transmission and hence it is added to the 24hour load demand data for scheduling.

6.5. Case Study 2. This system involves a standard Indian utility system with 19 units. All the 19 units include nonlinear characteristics such as valve point effect, ramp rate limit, multiple fuels, prohibited operating zones and spinning reserve constraint. This system is applied for three different load patterns as shown in Figure 5. The demand for each hour includes approximately $20 \%$ of transmission losses. A generation schedule is obtained using the DE-NM method where each unit contributes to the demand and the transmission loss. The maximum demand for pattern 1 is $4400 \mathrm{MW}$, for pattern 2 is $4186 \mathrm{MW}$, and for pattern 3 is $4173 \mathrm{MW}$. Table 3 gives the best generation schedule obtained by the DE-NM method for pattern 2. Few units in the system are provided with multiple fuel option. The fuel types include 1, 2, 3, 4, 5 


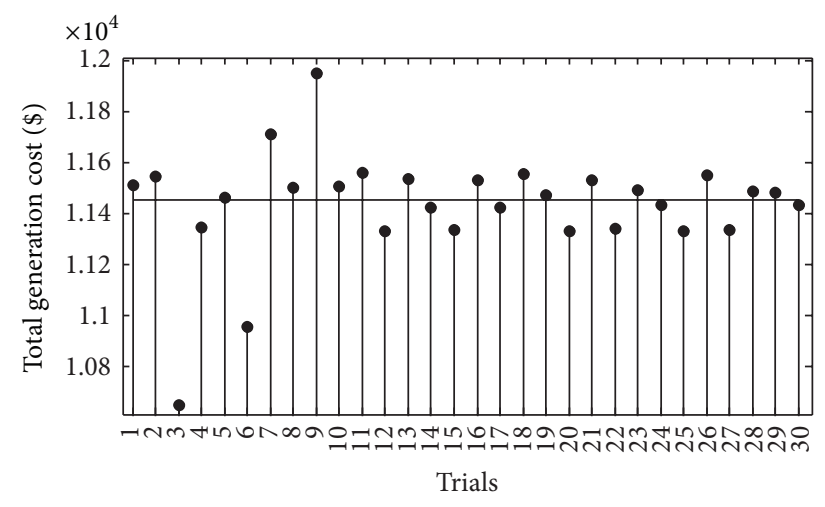

(a)

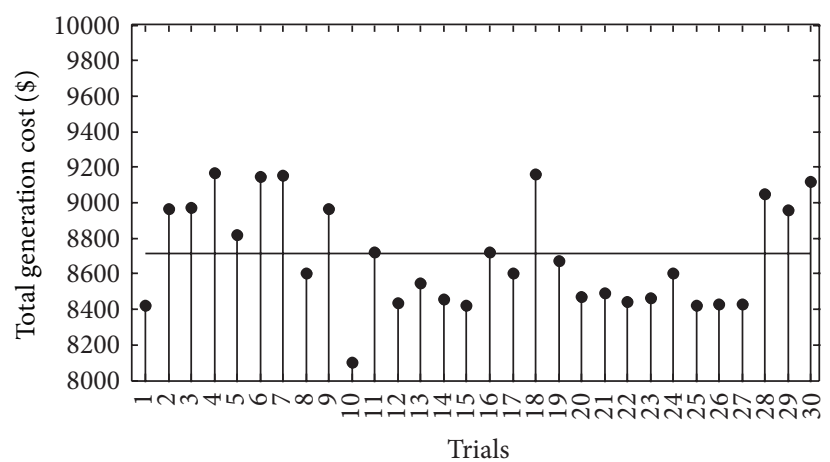

(b)

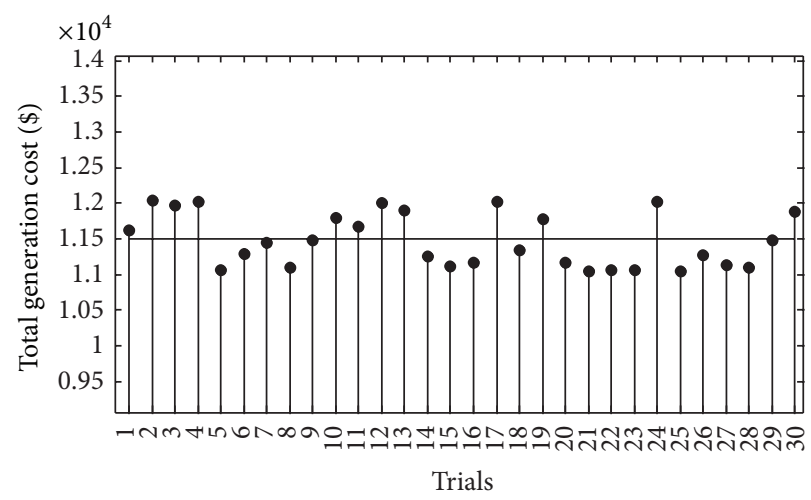

(c)

FIguRE 3: (a) Cost distribution obtained by DE-NM for case study 1 with pattern 1. (b) Cost distribution obtained by DE-NM for case study 1 with pattern 2. (c) Cost distribution obtained by DE-NM for case study 1 with pattern 3 .

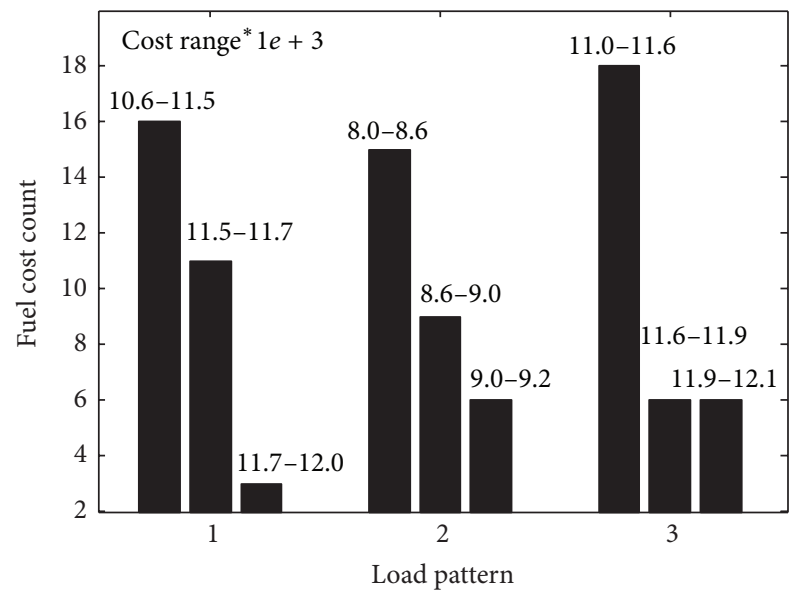

FIGURE 4: Frequency of convergence for case study 1.

and 6. Table 4 shows the fuel switching for units $3,5,7,13$, and 19 and all the remaining units utilize fuel 1 for every hour of time interval.

The best cost obtained by the DE-NM method for pattern 1,2 , and 3 is $\$ 404122.623, \$ 324962.343$, and $\$ 372140.528$, respectively. The worst cost for pattern 1 is $\$ 40565.659$, pattern 2 is $\$ 341440.818$, and pattern 3 is $\$ 374405.694$. Figures

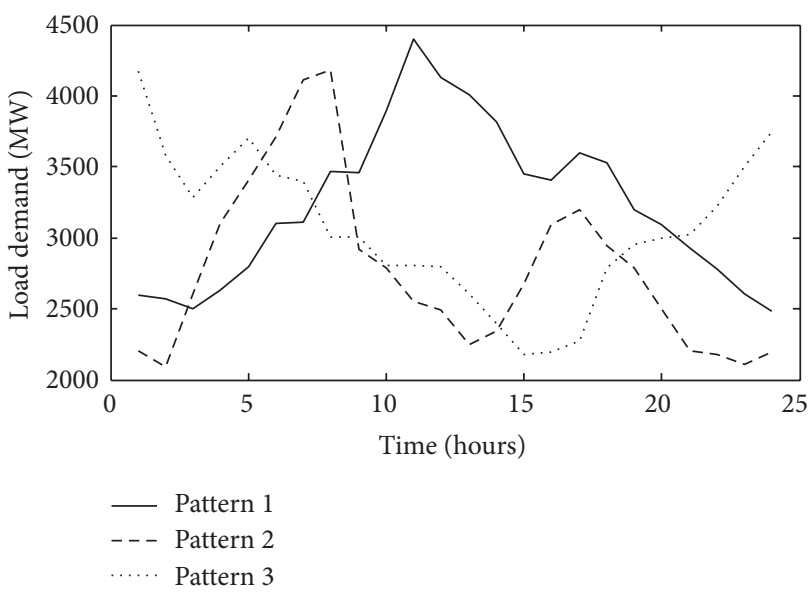

FIgURE 5: Load demand patterns for case study 2.

6(a), 6(b), and 6(c) shows the cost distribution for the system with pattern 1 , pattern 2 , and pattern 3 , respectively. The straight line running through the cost distribution area represents the average cost. The average cost for pattern 1 is 405515.7524 , pattern 2 is $\$ 334321.1071$, and pattern 3 is $\$ 33109.9977$. The percentage of producing quality solutions by DE-NM for the system is above $70 \%$ as seen from 
TABLE 3: Best power generation schedule obtained by DE-NM for case study 2.

\begin{tabular}{|c|c|c|c|c|c|c|c|c|c|c|}
\hline \multirow{2}{*}{ Hour } & \multicolumn{10}{|c|}{ Power output (MW) } \\
\hline & 1 & 2 & 3 & 4 & 5 & 6 & 7 & 8 & 9 & 10 \\
\hline 1 & 126.623 & 176.332 & 126.942 & 25.000 & 113.750 & 340.000 & 166.554 & 150.000 & 242.127 & 15.000 \\
\hline 2 & 124.189 & 165.000 & 144.530 & 08.000 & 146.176 & 340.000 & 177.498 & 138.874 & 254.528 & 15.000 \\
\hline 3 & 127.640 & 161.742 & 140.000 & 25.000 & 177.500 & 334.685 & 177.500 & 152.687 & 283.000 & 15.000 \\
\hline 4 & 132.015 & 287.062 & 368.136 & 25.000 & 175.995 & 340.000 & 177.499 & 165.069 & 260.000 & 40.000 \\
\hline 5 & 148.315 & 421.843 & 400.000 & 25.000 & 177.500 & 340.000 & 174.898 & 193.407 & 280.290 & 40.000 \\
\hline 6 & 227.936 & 438.000 & 440.000 & 25.000 & 177.500 & 340.000 & 176.290 & 321.681 & 302.381 & 40.000 \\
\hline 7 & 292.692 & 438.000 & 400.000 & 25.000 & 177.499 & 363.000 & 177.499 & 452.292 & 516.598 & 40.000 \\
\hline 8 & 300.000 & 438.000 & 400.000 & 25.000 & 177.500 & 371.145 & 177.499 & 500.000 & 532.465 & 40.000 \\
\hline 9 & 120.045 & 204.725 & 374.718 & 25.000 & 177.500 & 338.122 & 177.499 & 144.607 & 283.000 & 40.000 \\
\hline 10 & 129.676 & 196.189 & 202.088 & 25.000 & 177.500 & 340.000 & 177.500 & 153.581 & 257.593 & 40.000 \\
\hline 11 & 125.834 & 137.702 & 136.903 & 25.000 & 177.499 & 340.000 & 177.499 & 152.633 & 254.420 & 15.000 \\
\hline 12 & 126.297 & 149.825 & 140.000 & 25.000 & 177.497 & 335.679 & 177.484 & 294.088 & 238.824 & 15.000 \\
\hline 13 & 131.871 & 176.309 & 129.436 & 25.000 & 177.499 & 340.000 & 177.499 & 137.506 & 234.751 & 15.000 \\
\hline 14 & 119.616 & 177.057 & 103.519 & 25.000 & 177.499 & 340.000 & 177.499 & 142.830 & 260.000 & 15.000 \\
\hline 15 & 124.985 & 165.000 & 290.000 & 25.000 & 177.499 & 340.000 & 177.499 & 152.687 & 249.272 & 40.000 \\
\hline 16 & 114.362 & 309.274 & 337.442 & 25.000 & 177.499 & 340.000 & 177.499 & 152.334 & 239.253 & 40.000 \\
\hline 17 & 126.154 & 324.1193 & 377.603 & 25.000 & 177.499 & 340.000 & 177.499 & 158.274 & 254.610 & 40.000 \\
\hline 18 & 126.332 & 308.015 & 275.423 & 25.000 & 166.567 & 340.000 & 177.499 & 152.908 & 258.483 & 40.000 \\
\hline 19 & 125.370 & 187.353 & 288.980 & 25.000 & 177.499 & 340.000 & 177.499 & 123.743 & 260.000 & 15.000 \\
\hline 20 & 129.077 & 185.934 & 140.000 & 22.658 & 177.499 & 338.663 & 177.499 & 129.939 & 260.000 & 15.000 \\
\hline 21 & 103.540 & 154.576 & 301.215 & 25.000 & 167.760 & 332.659 & 177.500 & 149.915 & 267.546 & 15.000 \\
\hline 22 & 128.134 & 151.202 & 140.000 & 08.000 & 177.499 & 340.000 & 157.499 & 151.103 & 250.829 & 15.000 \\
\hline 23 & 118.221 & 165.000 & 140.000 & 08.000 & 177.499 & 331.385 & 168.524 & 147.996 & 291.043 & 15.000 \\
\hline 24 & 131.233 & 165.000 & 140.000 & 25.000 & 176.952 & 340.000 & 177.493 & 142.536 & 250.797 & 15.000 \\
\hline \multirow{2}{*}{ Hour } & \multicolumn{9}{|c|}{ Unit (MW) } & \\
\hline & 11 & 12 & 13 & 14 & 15 & 16 & 17 & 18 & 19 & \\
\hline 1 & 050.036 & 75.000 & 177.499 & 56.908 & 032.620 & 80.000 & 80.000 & 079.895 & 460.000 & \\
\hline 2 & 050.000 & 40.000 & 130.805 & 95.000 & 043.997 & 15.000 & 80.000 & 073.000 & 420.219 & \\
\hline 3 & 150.000 & 75.000 & 177.500 & 95.000 & 049.824 & 80.000 & 80.000 & 230.000 & 443.210 & \\
\hline 4 & 150.000 & 75.000 & 177.499 & 95.000 & 141.701 & 80.000 & 80.000 & 230.000 & 473.308 & \\
\hline 5 & 150.000 & 75.000 & 177.500 & 95.000 & 220.000 & 80.000 & 80.000 & 230.000 & 466.529 & \\
\hline 6 & 150.000 & 75.000 & 177.500 & 95.000 & 220.000 & 80.000 & 80.000 & 230.000 & 483.000 & \\
\hline 7 & 150.000 & 75.000 & 177.499 & 95.000 & 220.000 & 80.000 & 80.000 & 230.000 & 491.740 & \\
\hline 8 & 150.000 & 75.000 & 177.499 & 95.000 & 220.000 & 80.000 & 80.000 & 230.000 & 487.647 & \\
\hline 9 & 050.000 & 75.000 & 177.499 & 95.000 & 187.521 & 80.000 & 80.000 & 230.000 & 430.000 & \\
\hline 10 & 150.000 & 75.000 & 171.776 & 95.000 & 121.250 & 80.000 & 80.000 & 230.000 & 460.000 & \\
\hline 11 & 150.000 & 75.000 & 177.499 & 81.780 & 040.653 & 80.000 & 80.000 & 230.000 & 469.862 & \\
\hline 12 & 079.616 & 75.000 & 177.488 & 87.838 & 036.233 & 80.000 & 80.000 & 111.209 & 460.000 & \\
\hline 13 & 050.000 & 25.000 & 177.499 & 65.000 & 041.758 & 80.000 & 80.000 & 092.411 & 460.000 & \\
\hline 14 & 108.595 & 75.000 & 177.499 & 65.000 & 044.047 & 80.000 & 80.000 & 087.993 & 460.000 & \\
\hline 15 & 150.000 & 75.000 & 177.499 & 95.000 & 028.027 & 80.000 & 80.000 & 184.753 & 433.063 & \\
\hline 16 & 150.000 & 75.000 & 177.499 & 95.000 & 182.323 & 80.000 & 80.000 & 230.000 & 480.285 & \\
\hline 17 & 150.000 & 75.000 & 177.499 & 95.000 & 198.027 & 80.000 & 80.000 & 230.000 & 483.000 & \\
\hline 18 & 150.000 & 75.000 & 177.499 & 95.000 & 094.367 & 80.000 & 80.000 & 230.000 & 466.193 & \\
\hline 19 & 150.000 & 75.000 & 177.499 & 95.000 & 091.068 & 80.000 & 80.000 & 230.000 & 464.949 & \\
\hline 20 & 150.000 & 75.000 & 177.499 & 31.432 & 035.402 & 80.000 & 80.000 & 230.000 & 438.682 & \\
\hline 21 & 060.000 & 75.000 & 113.750 & 65.000 & 044.040 & 80.000 & 80.000 & 073.000 & 460.000 & \\
\hline 22 & 150.000 & 75.000 & 112.426 & 35.000 & 044.592 & 80.000 & 80.000 & 073.000 & 380.409 & \\
\hline 23 & 50.000 & 75.000 & 113.750 & 94.352 & 033.301 & 15.000 & 44.215 & 073.000 & 460.000 & \\
\hline 24 & 50.000 & 75.000 & 177.499 & 95.000 & 044.034 & 45.000 & 16.283 & 050.000 & 454.875 & \\
\hline
\end{tabular}




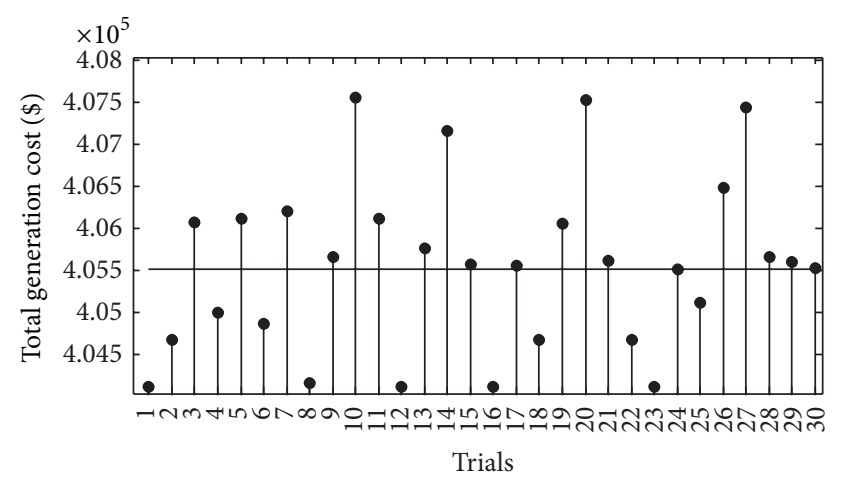

(a)

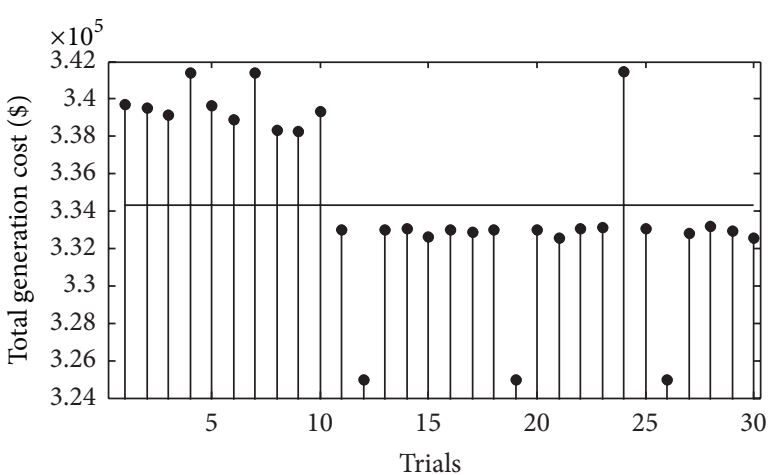

(b)

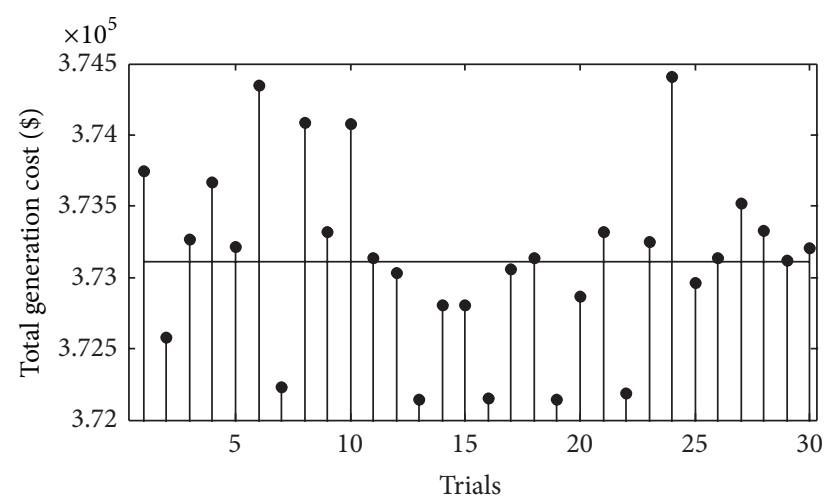

(c)

FIGURE 6: (a) Cost distribution obtained by DE-NM for case study 2 with pattern 1. (b) Cost distribution obtained by DE-NM for case study 2 with pattern 2. (c) Cost distribution obtained by DE-NM for case study 2 with pattern 3.

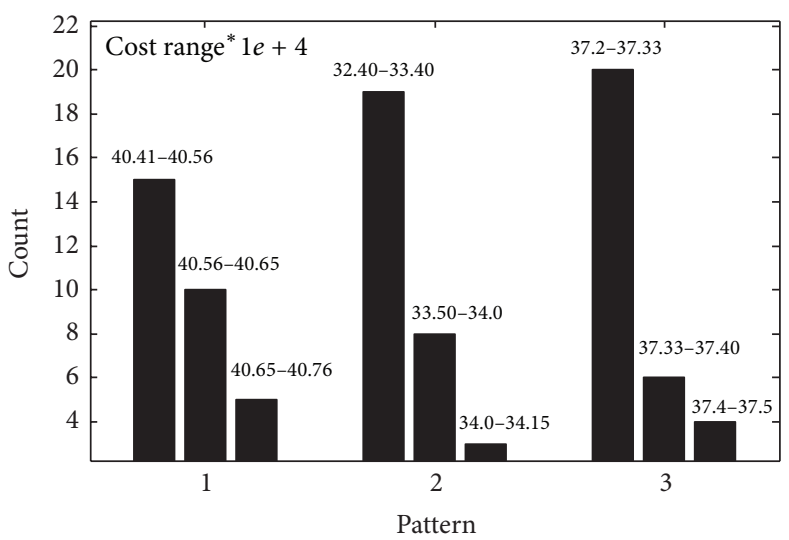

Figure 7: Frequency of convergence for case study 2.

Figure 7. Table 5 clearly shows the computational time of the three patterns for both the test systems. Table 6 presents the relationship between $k$, computation time, and result quality for a 10-unit system.

\section{Conclusion}

A maiden attempt has been taken to apply Differential Evolution with Neighborhood based Mutation (DE-NM) method to solve Dynamic Economic Dispatch (DED) problem including ramp rate effects, prohibited operating zones, spinning reserve, and multiple fuel options under a single frame. To show the effectiveness of the DE-NM method, an Indian utility system and a 10 -unit system are presented to solve the DED problem for a given load profile. The results show that DE-NM is efficient in handling the constraints and it is applicable to larger systems. Due to enormous transmission lines in the Indian utility system, a proper model 
TABLE 4: Fuel switching of generators for case study 2.

\begin{tabular}{lccccc}
\hline \multirow{2}{*}{ Hour } & \multicolumn{5}{c}{ Unit (MW) } \\
& 3 & 5 & 7 & 13 & 19 \\
\hline 1 & $1,1,2$ & $2,4,2$ & $2,6,2$ & $2,2,2$ & $1,1,2$ \\
2 & $2,1,2$ & $2,4,2$ & $2,2,2$ & $2,1,2$ & $1,1,1$ \\
3 & $1,1,2$ & $2,2,2$ & $2,2,2$ & $2,2,2$ & $1,1,1$ \\
4 & $1,2,2$ & $2,2,2$ & $2,2,2$ & $6,2,2$ & $1,1,1$ \\
5 & $2,2,2$ & $2,2,2$ & $2,2,2$ & $2,2,2$ & $1,1,1$ \\
6 & $2,2,2$ & $2,2,2$ & $2,2,2$ & $2,2,2$ & $1,1,1$ \\
7 & $2,2,2$ & $2,2,2$ & $6,2,2$ & $2,2,6$ & $1,1,1$ \\
8 & $2,2,2$ & $2,2,2$ & $2,2,2$ & $2,2,2$ & $2,1,1$ \\
9 & $2,2,2$ & $2,2,2$ & $2,2,2$ & $2,2,2$ & $1,1,1$ \\
10 & $2,1,2$ & $2,2,2$ & $2,2,2$ & $2,2,2$ & $1,1,1$ \\
11 & $2,1,2$ & $2,2,2$ & $2,2,2$ & $2,2,2$ & $2,1,1$ \\
12 & $2,1,2$ & $2,2,2$ & $2,2,6$ & $2,2,2$ & $2,1,1$ \\
13 & $2,1,1$ & $2,2,2$ & $2,2,2$ & $2,2,2$ & $1,1,1$ \\
14 & $2,1,1$ & $2,2,2$ & $2,2,2$ & $2,2,2$ & $2,1,1$ \\
15 & $2,2,1$ & $2,2,2$ & $2,2,2$ & $2,2,2$ & $1,1,1$ \\
16 & $2,2,1$ & $2,2,2$ & $2,2,2$ & $2,2,2$ & $2,1,1$ \\
17 & $2,2,1$ & $2,2,2$ & $2,2,2$ & $2,2,1$ & $2,1,1$ \\
18 & $2,2,2$ & $2,6,2$ & $2,2,6$ & $2,2,2$ & $1,1,1$ \\
19 & $2,2,2$ & $2,2,2$ & $2,2,2$ & $2,2,2$ & $1,1,1$ \\
20 & $2,1,2$ & $2,2,2$ & $2,2,2$ & $2,2,2$ & $1,1,1$ \\
21 & $2,2,2$ & $2,2,2$ & $2,2,2$ & $2,1,2$ & $1,1,1$ \\
22 & $2,1,2$ & $2,2,2$ & $2,6,6$ & $6,1,2$ & $1,1,1$ \\
23 & $1,1,2$ & $2,2,2$ & $2,6,2$ & $2,1,2$ & $1,1,1$ \\
24 & $1,1,2$ & $2,2,2$ & $2,2,2$ & $2,2,2$ & $1,1,1$ \\
\hline & & & & &
\end{tabular}

TABLE 5: Computation time for both systems for 1000 iterations with a population size of 100 .

\begin{tabular}{|c|c|c|c|}
\hline \multicolumn{2}{|c|}{ 10-unit system } & \multicolumn{2}{|c|}{ 19-unit system } \\
\hline Load pattern & $\mathrm{Sec}$ & Load pattern & $\mathrm{Sec}$ \\
\hline 1 & 13.581 & 1 & 32.939 \\
\hline 2 & 12.121 & 2 & 33.782 \\
\hline 3 & 11.465 & 3 & 34.566 \\
\hline
\end{tabular}

TABLE 6: Relationship between $k$, computation time, and result quality for a 10-unit system.

\begin{tabular}{cccc}
\hline & $k$ & Time in Sec & Cost range \\
\hline \multirow{3}{*}{ Pattern 1 } & $10 \%$ & 13.581 & $11640-11500$ \\
& $20 \%$ & 96.32 & $11583-11472$ \\
& $30 \%$ & 1134.15 & $11953-11678$ \\
\hline \multirow{3}{*}{ Pattern 2 } & $10 \%$ & 12.121 & $8600-8000$ \\
& $20 \%$ & 89.56 & $8578-7926$ \\
\multirow{2}{*}{ Pattern 3 } & $30 \%$ & 985.23 & $8927-8712$ \\
& $10 \%$ & 11.465 & $11600-11000$ \\
& $20 \%$ & 73.42 & $11578-11215$ \\
& $30 \%$ & 921.73 & $11827-11749$ \\
\hline
\end{tabular}

for including the losses is still under the search. The search ability of the DE-NM is improved by striking proper balance between exploration and exploitation process. The simulation results show that the DE-NM method is capable of producing solutions which are near optimal and has stable converging characteristics.

\section{Nomenclature}

$F_{T}: \quad \quad \quad$ Total fuel cost of the system (\$)

$F_{i h}\left(P_{i h}\right): \quad$ Incremental fuel cost function $(\$ / \mathrm{h})$

$P_{i h}: \quad$ Real power output of the $i$ th unit at the $h$ th interval (MW)

$N: \quad \quad$ Number of generating units

$H: \quad$ Number of intervals in the given time period

$a_{i}, b_{i}$, and $c_{i}$ : Cost coefficients of the $i$ th generating unit

$e_{i}$ and $f_{i}$ : Constants from the valve point of the $i$ th generating unit

$P_{i h}^{\min } / P_{i h}^{\max }:$ Minimum/maximum limit of the real power of the $i$ th unit at $h$ th interval (MW)

$N_{F}$ : Number of fuel options for each generating unit

$P_{D h}: \quad$ Power demand at the $h$ th interval (MW)

$P_{\text {loss }, h}: \quad$ Power loss at the $h$ th interval (MW)

$B_{m n}: \quad$ Transmission loss coefficients

$\mathrm{UR}_{i} / \mathrm{DR}_{i}: \quad \mathrm{Up} /$ down ramp rate limits of the $i$ th unit (MW)

$P_{i(h-1)}$ : $\quad$ Power generated by the $i$ th unit at the $(h-1)$ th interval (MW)

$n: \quad$ Number of generators with prohibited operating zones

$P_{i, n}^{u} / P_{i, n}^{l}: \quad$ Upper/lower limit of the $n$th prohibited zone for $i$ th generating unit

$S_{i h}: \quad$ Spinning reserve contribution of the $i$ th generating unit at the $h$ th interval (MW)

$S_{R}: \quad$ System spinning reserve requirement (MW)

$S_{i}^{\max }$ : Maximum spinning reserve contribution of the $i$ th generating unit (MW)

NP: $\quad$ Total number of population

$\alpha$ and $\beta$ : Scaling factors

$w: \quad$ Scalar weight

$G_{\max }: \quad$ Maximum generation count

Cr: $\quad$ Crossover rate.

\section{Conflict of Interests}

The authors declare that there is no conflict of interests regarding the publication of this paper.

\section{Acknowledgment}

The authors would like to thank the Executive Engineer Mr. S. Murugesan for his kind technical support and data provided for implementing this algorithm in 19-unit Indian utility system.

\section{References}

[1] B. H. Chowdhury and S. Rahman, "A review of recent advances in economic dispatch," IEEE Transactions on Power Systems, vol. 5, no. 4, pp. 1248-1259, 1990. 
[2] X. S. Han and H. B. Gooi, "Effective economic dispatch model and algorithm," International Journal of Electrical Power and Energy Systems, vol. 29, no. 2, pp. 113-120, 2007.

[3] C. Wang and S. M. Shahidehpour, "Effects of ramp-rate limits on unit commitment and economic dispatch," IEEE Transactions on Power Systems, vol. 8, no. 3, pp. 1341-1450, 1993.

[4] G. P. Granelli, P. Marannino, M. Montagna, and A. Silvestri, "Fast and efficient gradient projection algorithm for dynamic generation dispatching," IEE Proceedings C, vol. 136, no. 5, pp. 295-302, 1989.

[5] K. S. Hindi and M. R. Ab Ghani, "Dynamic economic dispatch for large scale power systems: a Lagrangian relaxation approach," International Journal of Electrical Power and Energy Systems, vol. 13, no. 1, pp. 51-56, 1991.

[6] F. N. Lee, L. Lemonidis, and K. Liu, "Price-based ramp-rate model for dynamic dispatch and unit commitment," IEEE Transactions on Power Systems, vol. 9, no. 3, pp. 1233-1242, 1994.

[7] D. L. Travers and R. J. Kaye, "Dynamic dispatch by constructive dynamic programming," IEEE Transactions on Power Systems, vol. 13, no. 1, pp. 72-78, 1998.

[8] D. C. Walters and G. B. Sheble, "Genetic algorithm solution of economic dispatch with value point loading," IEEE Transactions on Power Systems, vol. 8, no. 3, pp. 1325-1332, 1993.

[9] A. I. Selvakumar and K. Thanushkodi, "Anti-predatory particle swarm optimization: solution to nonconvex economic dispatch problems," Electric Power Systems Research, vol. 78, no. 1, pp. 210, 2008.

[10] S. Titus and A. E. Jeyakumar, "A hybrid EP-PSO-SQP algorithm for dynamic dispatch considering prohibited operating zones," Electric Power Components and Systems, vol. 36, no. 5, pp. 449467, 2008.

[11] P. P. J. van den Bosch, "Optimal dynamic dispatch owing to spinning-reserve and power-rate limits," IEEE Transactions on Power Apparatus and Systems, vol. 104, no. 12, pp. 3395-3401, 1985.

[12] A. H. Rashed, E. N. Abdallah, and F. Benhamida, "A hopfield neural network based dynamic dispatch," in Proceedings of the 10th International Middle East Power System Conference, pp. 515-525, December 2005.

[13] H. Yang, P. Yang, and C. Huang, "Evolutionary programming based economic dispatch for units with non-smooth fuel cost functions," IEEE Transactions on Power Systems, vol. 11, no. 1, pp. 112-118, 1996.

[14] C. K. Panigrahi, P. K. Chattopadhyay, R. N. Chakrabarti, and M. Basu, "Simulated annealing technique for dynamic economic dispatch," Electric Power Components and Systems, vol. 34, no. 5, pp. 577-586, 2006.

[15] T. A. A. Victoire and A. E. Jeyakumar, "Deterministically guided PSO for dynamic dispatch considering valve-point effect," Electric Power Systems Research, vol. 73, no. 3, pp. 313-322, 2005.

[16] R. Balamurugan and S. Subramanian, "Differential evolutionbased dynamic economic dispatch of generating units with valve-point effects," Electric Power Components and Systems, vol. 36, no. 8, pp. 828-843, 2008.

[17] P. Chakraborty, G. G. Roy, B. K. Panigrahi, R. C. Bansal, and A. Mohapatra, "Dynamic economic dispatch using harmony search algorithm with modified differential mutation operator," Electrical Engineering, vol. 94, no. 4, pp. 197-205, 2012.

[18] Y. Chen, J. Wen, L. Jiang, and S. Cheng, "Hybrid algorithm for dynamic economic dispatch with valve-point effects," IET Generation, Transmission and Distribution, vol. 7, no. 10, pp. 1096-1104, 2013.
[19] X. Xia and A. M. Elaiw, "Optimal dynamic economic dispatch of generation: a review," Electric Power Systems Research, vol. 80, no. 8, pp. 975-986, 2010.

[20] T. A. A. Victoire and A. E. Jeyakumar, "A modified hybrid EPSQP approach for dynamic dispatch with valve-point effect," International Journal of Electrical Power and Energy Systems, vol. 27, no. 8, pp. 594-601, 2005.

[21] T. A. A. Victoire and A. E. Jeyakumar, "Hybrid PSO-SQP for economic dispatch with valve-point effect," Electric Power Systems Research, vol. 71, no. 1, pp. 51-59, 2004.

[22] L. D. S. Coelho and V. C. Mariani, "Combining of chaotic differential evolution and quadratic programming for economic dispatch optimization with valve-point effect," IEEE Transactions on Power Systems, vol. 21, no. 2, pp. 989-996, 2006.

[23] J. Liu and J. Lampinen, "On setting the control parameters of the differential evolution method," in Proceedings of the 8th International Conference on Soft Computing, pp. 11-18, Brno, Czech Republic, June 2002.

[24] A. Salman, A. P. Engelbrecht, and M. G. H. Omran, "Empirical analysis of self-adaptive differential evolution," European Journal of Operational Research, vol. 183, no. 2, pp. 785-804, 2007.

[25] R. S. Rahnamayan, H. R. Tizhoosh, and M. M. A. Salama, "Opposition-based differential evolution," IEEE Transactions on Evolutionary Computation, vol. 12, no. 1, pp. 64-79, 2008.

[26] N. Noman and H. Iba, "Accelerating differential evolution using an adaptive local search," IEEE Transactions on Evolutionary Computation, vol. 12, no. 1, pp. 107-125, 2008.

[27] S. Das, A. Abraham, U. K. Chakraborty, and A. Konar, "Differential evolution using a neighborhood-based mutation operator," IEEE Transactions on Evolutionary Computation, vol. 13, no. 3, pp. 526-553, 2009.

[28] K. Price, R. Storn, and J. Lampinen, Differential Evolution: A Practical Approach to Global Optimization, Springer, Berlin, Germany, 2005.

[29] U. K. Chakraborty, S. Das, and A. Konar, "Differential evolution with local neighborhood," in Proceedings of the IEEE Congress on Evolutionary Computation (CEC '06), pp. 2042-2049, Vancouver, Canada, July 2006.

[30] C. Chiang, "Improved genetic algorithm for power economic dispatch of units with valve-point effects and multiple fuels," IEEE Transactions on Power Systems, vol. 20, no. 4, pp. 1690$1699,2005$. 


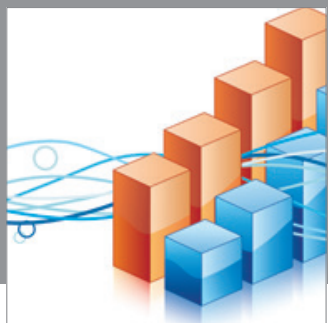

Advances in

Operations Research

mansans

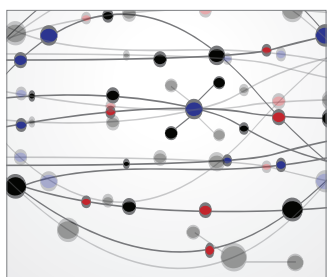

The Scientific World Journal
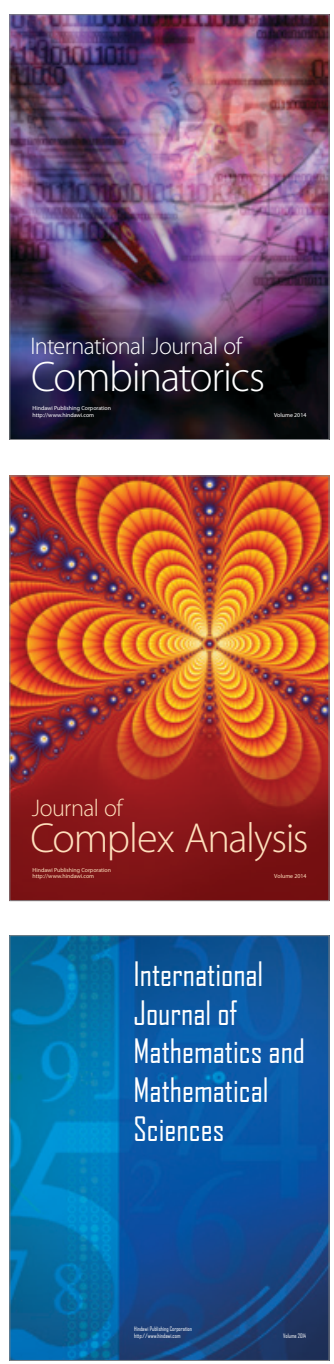
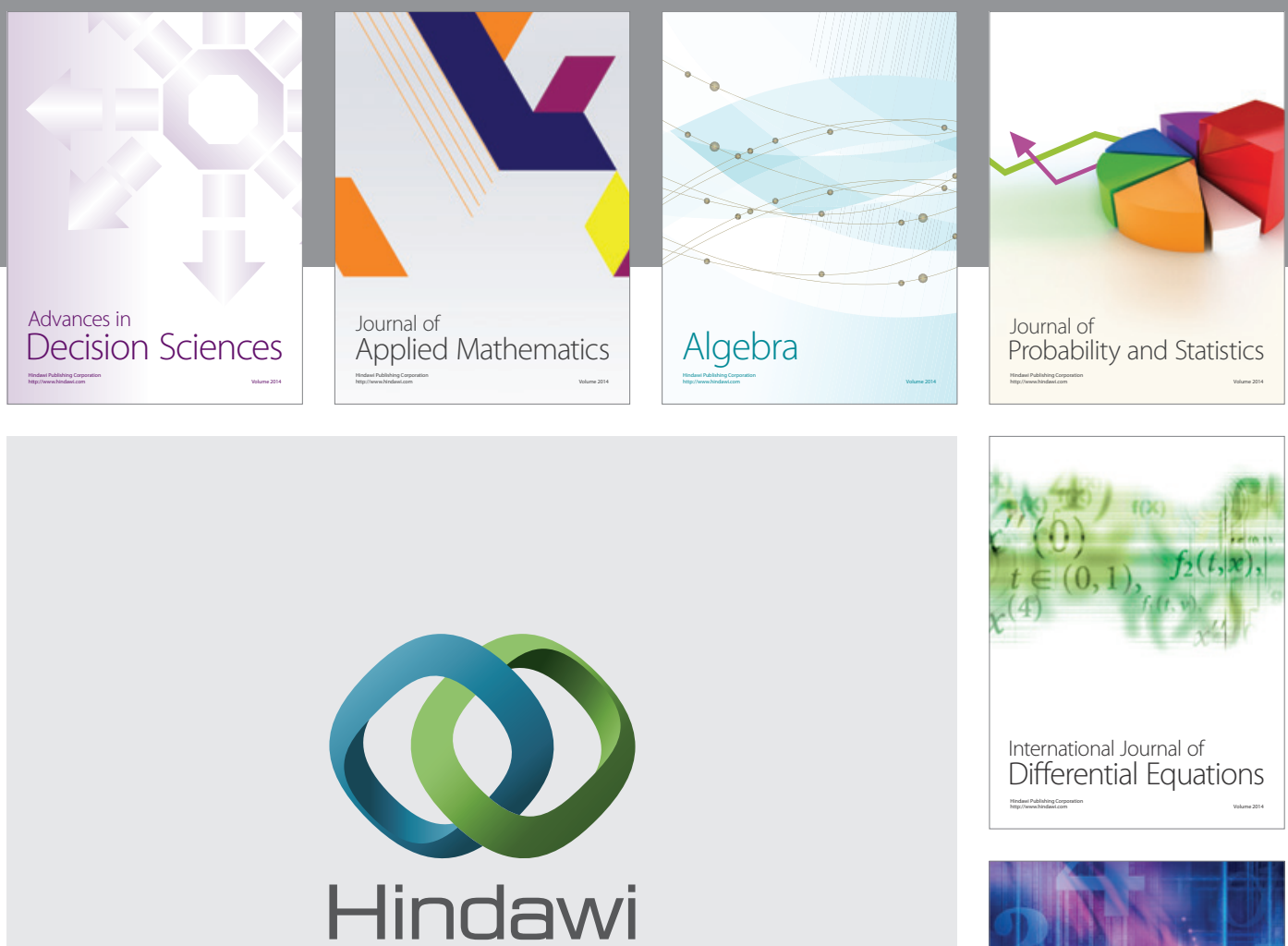

Submit your manuscripts at http://www.hindawi.com
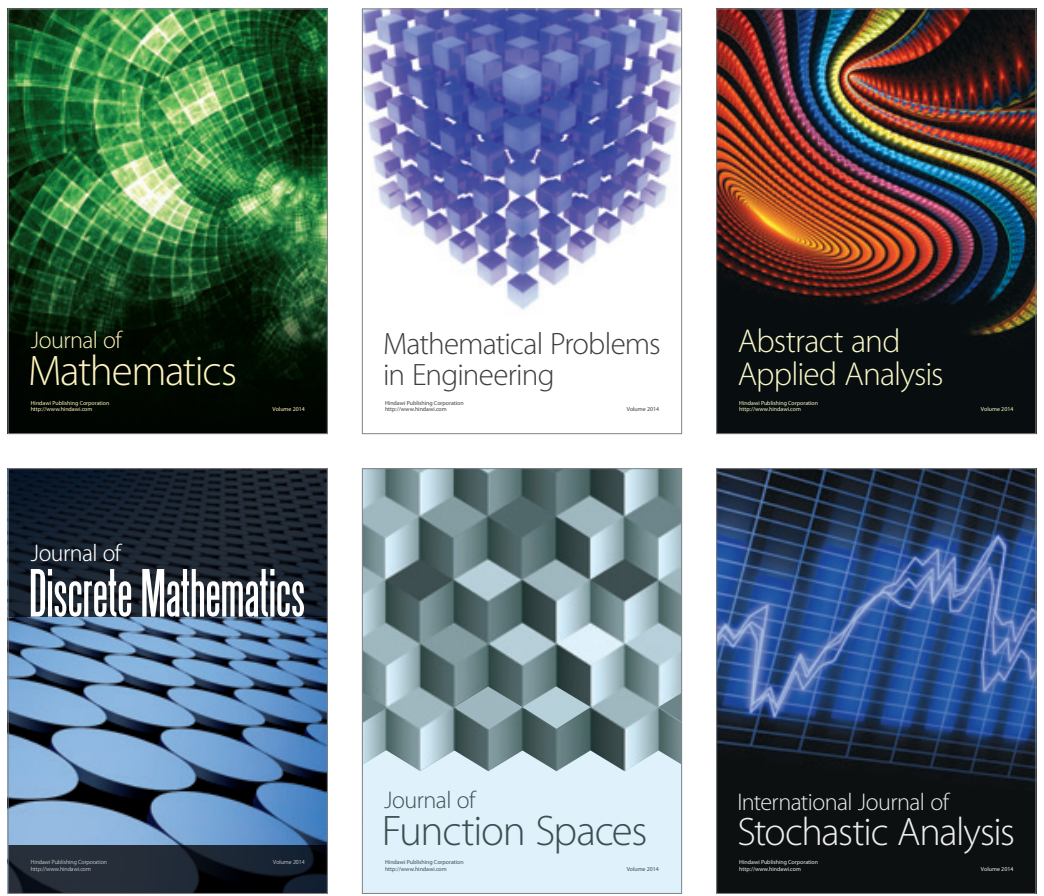

Journal of

Function Spaces

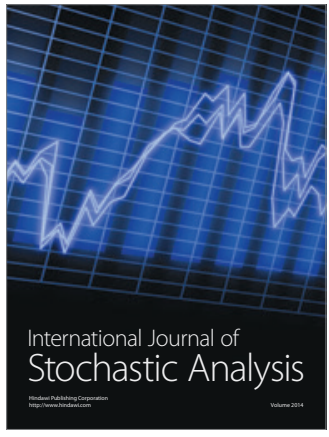

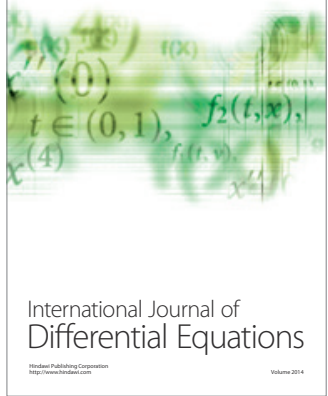
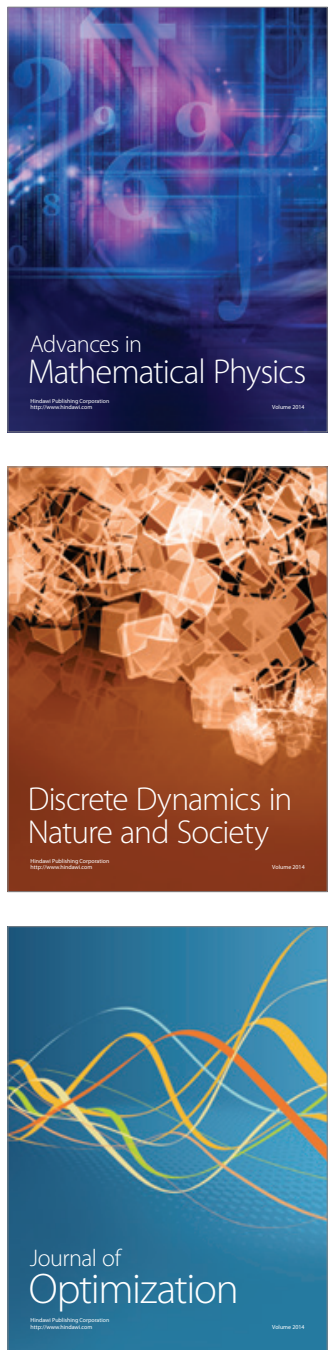